\title{
Transatlantica
}

Revue d'études américaines. American Studies Journal

\section{Mary Cassatt au Mona Bismarck American Center for Arts and Culture, et Paul Sietsema à la Drawing Room, Londres : images re-produites, visions fractionnées.}

\section{Kamila Benayada}

\section{OpenEdition}

\section{Journals}

Édition électronique

URL : https://journals.openedition.org/transatlantica/6112

DOI : $10.4000 /$ transatlantica.6112

ISSN : 1765-2766

Éditeur

Association française d'Etudes Américaines (AFEA)

Référence électronique

Kamila Benayada, "Mary Cassatt au Mona Bismarck American Center for Arts and Culture, et Paul Sietsema à la Drawing Room, Londres : images re-produites, visions fractionnées. », Transatlantica [En ligne], 2 | 2012, mis en ligne le 02 mai 2013, consulté le 06 avril 2023. URL : http:// journals.openedition.org/transatlantica/6112 ; DOI : https://doi.org/10.4000/transatlantica.6112

Ce document a été généré automatiquement le 6 avril 2023.

Creative Commons - Attribution - Pas d'Utilisation Commerciale - Pas de Modification 4.0 International - CC BY-NC-ND 4.0

https://creativecommons.org/licenses/by-nc-nd/4.0/ 


\title{
Mary Cassatt au Mona Bismarck American Center for Arts and Culture, et Paul Sietsema à la Drawing Room, Londres : images re- produites, visions fractionnées.
}

\author{
Kamila Benayada
}

1 Les dessins et gravures de Mary Cassatt (1844-1926), de la collection de son marchand Ambroise Vollard, étaient exposés à Paris jusqu'au 20 janvier dernier. Au même moment, du 20 septembre au 10 novembre 2012, la nouvelle galerie londonienne « Drawing Room» montrait le travail de Paul Sietsema (1968 -). Plus d'un siècle sépare ces deux artistes; pourtant, les œuvres présentées, malgré leurs différences, soulevaient des questions esthétiques analogues.

2 Mary Cassatt peint et dessine la vie domestique des femmes de son entourage. D'une famille aisée, elle étudie à la Philadelphia School of Fine Arts, puis en Italie, et enfin en France, sous Gerôme. Ayant rencontré Degas en 1874, elle s'initie avec lui à la gravure et découvre avec la presse de l'artiste impressionniste la multiplicité des copies et l'inconstance des états. L'exposition révèle cette progression de l'épreuve initiale vers autre chose, à travers une exploration des multiples possibilités offertes par la reproduction, alors que l'éventail des sujets possibles qui s'offraient à une femme artiste restait très limité. C'est donc moins le sujet qui ouvre la porte à la créativité, que le médium ainsi que la technique de la reproduction. Cassatt représente des scènes de la vie quotidienne, le monde clos des épouses et des mères, passant de la loge d'opéra au bain des enfants ou à l'intérieur du tramway. Sa peinture murale Modern Woman (1893) montre des femmes dans un jardin, cueillant les fruits d'un savoir qui reste le produit d'un monde privé. Ce qu'il y a au-delà du mur est inaccessible et la composition ramène le regard vers ce qui se passe à l'intérieur du jardin et vers la surface de l'œuvre. 
3 L'exposition présente le cheminement de l'artiste, à travers les différents états de son travail, c'est-à-dire à la fois ses essais sur la couleur et la reprise du tracé des lignes, en finesse ou en épaisseur. Le dessin initial demeure, mais ces variations ne cessent de modifier l'œuvre. Ainsi, l'altérité vient non pas du sujet, mais de son traitement, de la forme plutôt que du fond, de l'intérieur plutôt que de l'extérieur (lignes et masse dans le travail en noir et blanc, couleurs après la découverte des estampes japonaises en 1889). Une salle entière dédiée aux pastels montre le travail de Cassatt sur la contreépreuve, le transfert du dessin au pastel sur une feuille de papier de riz détrempée sur l'envers, où l'original est reproduit inversé, reconnaissable mais autre, encore une fois, sans jamais que l'artiste ne retouche ces images-miroir (à l'inverse de son ami Degas). L'imperfection du double importe donc moins à Cassatt que le processus du transfert ou de transformation d'un produit fini. Comme plus tard Stuart Davis, Cassatt travaille en circuit clos : sa créativité s'exprime dans la manipulation technique des médiums, et dans le travail manuel, artisanal de la reproduction. La progression non-linéaire du travail tel qu'il est présenté au centre Mona Bismarck, où les épreuves sont mises côte à côte sans aucune numérotation ne gênent nullement l'appréciation des œuvres mais suggère au contraire une appréhension moderne du temps, où la juxtaposition et la superposition l'emportent sur la succession. L'œuvre est ainsi plurielle, faite des succès et des ratés d'une composition sur un même thème ou sujet (lignes trop épaisses, bavures, aplats de couleurs imprécis), voire de la totalité du travail de l'artiste.

En tout cela, Mary Cassatt annonce le XX ${ }^{\text {ème }}$ siècle et le modernisme américain. Mais aussi le XXI ${ }^{\mathrm{ème}}$ siècle, et Paul Sietsema.

Comme Cassatt, mais pour d'autres raisons, Sietsema peint l'intériorité à travers le monde clos de son atelier. Il accorde une grande importance au dessin, ce qui motive cette exposition dans un lieu consacré avant tout aux œuvres sur papier. Partant du dessin, il se dirige dans d'autres directions, telles que la peinture et la vidéo. Son travail, a pour point de départ son environnement (atelier, bibliothèque) et ce qui s'y trouve. Il reprend des articles de journaux en en redessinant chaque détail, colle la photographie numérique d'une brosse ou d'outils sur une toile sur laquelle il fait couler de la peinture (Painting for Assembly, 2012, ou Brush painting, 2012). Dans ces deux œuvres (et ailleurs), il utilise des toiles de peintres aujourd'hui inconnus et les peint retendues sur l'envers, gardant les traces de salissures. Par ailleurs, il photographie les clous et les outils de l'encadreur et en inclut l'image dans son nouveau tableau. Double et original, peinture et photographie, passé et présent s'entremêlent pour mieux tromper le regard. Sietsema mixe des techniques passées de mode et innovations technologiques dans le but de placer son œuvre hors de l'époque où elles sont produites.

6 Son intérêt pour les voiliers, qu'il reproduit dans la série des Calendar Boats (2012) vient de ce qu'ils représentent «a kind of non-space of achronic time, not past not present, not future $»^{1}$. Mais la série elle-même est hors du temps, car non seulement il est impossible de différencier les divers voiliers mais le dessin lui-même ressemble à s'y méprendre à une photographie. Sietsema joue avec notre regard, en datant les voiliers/ dessins (de 2010 à 2013), de sorte que présent et passé ne se distinguent plus que par de subtiles variations de couleur. Donnée variable chez les deux artistes, le temps, chez Cassatt comme chez Sietsema échappe à son inéluctable linéarité par le biais de la multiplicité des versions proposées, qui disséminent le regard. Dans les Calendar Boats, Sietsema reproduit sous forme éclatée puis recomposée la photographie d'un voilier, 
dont l'image (qui n'est qu'un cliché) compte moins que la technique de sa (re)production.

7 Sietsema se filme aussi à l'œuvre, produisant des sculptures à partir d'objets trouvés. Comme Cassatt avant lui, il s'intéresse aux «non-œuvres »: Cassatt en gardant toutes les épreuves et états de ses gravures, réussies ou non, achevées ou non, ou en ne retouchant pas ses contre-épreuves de pastels, Sietsema en reprenant les objets du quotidien, s'interrogeant ainsi sur ce qui est suceptible de faire œuvre. Dans Blue Square I, et Blue Square 2 (2012), il sépare le cadre de ce qui est encadré : dans la première partie de l'œuvre, il reproduit à l'encre un carré de papier bleu froissé, déchiré, écorné, et dans la deuxième partie, il encadre les morceaux de bois qui pourraient constituer un cadre. Il reproduit ainsi la matière de l'encadrement, qui fonctionne aussi comme médium d'un travail sur la ligne et la géométrie. Matière et médium se superposent et se complètent tout en s'opposant. Sietsema s'intéresse à la tension entre matière et langage qui peut révéler les limites ou le potentiel non exploité du langage, tension qui renvoie à la communication numérique où il n'y a aucune relation entre le support et le message $^{2}$. Son travail explore le mode d'appréhension du regard et de la compréhension de l'œuvre à travers la reproduction sous différentes formes, et ses liens avec la transposition inhérente aux systèmes de reproduction (photographie, copie, peinture, vidéo).

Cassatt reproduit (pour ensuite s'en échapper) une matrice culturelle qui place les femmes dans un monde spécifique, reproduit l'enfermement, mais ramène le regard à la surface des œuvres, en neutralisant l'arrière-plan que lui impose ses sujets (mur de jardin, fenêtre de tramway, arrière de loge). Son travail sur la duplication et le renversement de l'image sont autant de détournements du sujet au profit de la forme, qui questionnent le regard.

9 Sietsema de son côté reproduit des images dont l'original est le résultat de techniques mécaniques ou numériques et de matériaux très divers, en s'intéressant à la relation entre ces différents paramètres ainsi qu'à la relation entre image et arrière-plan, matière et sens. De là, il explore tout à la fois le rôle de l'artiste dans la production culturelle, la production de matrices culturelles à partir d'objets (artistiques ou usuels), la place accordée aux objets (artistiques et usuels) dans la culture ainsi que la construction d'un savoir à partir de systèmes de compréhension. Dans un entretien avec Carter Mull, il déclarait ainsi: "I think I'm trying to get at somethingspecific about representation, something about structures and reception vs. depiction ${ }^{3}$. Les deux artistes destructurent l'œuvre, invitant le spectateur à suivre Sietsema, dans une analyse des modes de réception qui remet en question la représentation elle-même.

\section{NOTES}

1. Paul Sietsema, entretien avec Kate Macfarlane, commissaire de l'exposition, guide de l'exposition, www.drawingroom.org.uk/exhibitions/paul_sietsema.php 
2. L'artiste explique, au sujet de sa nouvelle video Telegraph (2012) qu'il s'intéresse à « ... how an agent like this can work against language, or linguistic thought, and perhaps expose the limits (and perhaps untapped potentials) of language. But of course also the ambiguousness of a piece of wood parallels the formless ambiguity of the mechanism of digital communication, the 0 and the 1 , the rootlessness of the kind of non-relationship of the carrier and the message ", ibid.

3. Paul Sietsema, in 'Paul Sietsema Interview with Carter Mull', Marcel \# 3, Sept. 2010, reproduit dans le guide de l'exposition.

INDEX

Thèmes : Trans'Arts

\section{AUTEUR}

\section{KAMILA BENAYADA}

Université d'Orléans 